\title{
Emergency nurses' strategies in addressing uncertainty and change in the management of emerging infectious diseases: A grounded theory approach
}

Stanley Kam Ki Lam ( $\sim$ stanleylam@twc.edu.hk )

Tung Wah College https://orcid.org/0000-0002-6544-795X

Enid Wai Yung Kwong

Hong Kong Polytechnic University

Maria Shuk Yu Hung

Tung Wah College

Samantha Mei Che Pang

Hong Kong Polytechnic University

Wai Tong Chien

Chinese University of Hong Kong

\section{Research article}

Keywords: Emerging infectious diseases, Emergency nurses, Nursing, Uncertainty, Change, Qualitative study

Posted Date: September 5th, 2019

DOI: https://doi.org/10.21203/rs.2.13920/v1

License: (c) (i) This work is licensed under a Creative Commons Attribution 4.0 International License.

Read Full License 


\section{Abstract}

Background Uncertainty and change are the inevitable challenges facing emergency nurses, as they are bound by the nature of emergency care. During an epidemic event, emergency care settings are intertwined with urgency, uncertainty, and change, which continuously pose challenges to emergency nurses on their capacity to act on their duties in a well-planned and systematic manner. However, there has been a paucity of understanding concerning emergency nurses' actions and strategies to address the instabilities and vulnerabilities of the circumstance. The present study aimed to explore emergency nurses' behaviours and strategies in addressing the challenges of uncertainty and change during an epidemic event.

Methods A qualitative design based upon a Straussian grounded theory approach was selected as the method of inquiry. A total of 26 emergency nurses from Hong Kong were recruited in the study by purposive and theoretical sampling strategies. Semi-structured, face-to-face, individual interviews were conducted for data collection. The data were transcribed verbatim and analysed through the grounded theory coding procedures. The COREQ guidelines were applied in the reporting of the present study.

Results Rehearsing for improvisation was revealed as the core category. Four interplaying sub-categories were identified: (a) sensing the unclear situation, (b) equipping for the impending battle, (c) calibrating to the evolving guidelines, and (d) navigating the new role and duties. The nurses demonstrated their prudence to orientate themselves to an ambiguous work situation and displayed the flexibility to embrace changes in their practice, roles, and duties.

Conclusions The findings delineate that emergency nurses were required to prepare and equip themselves with skills and strategies to improvise and adapt to the management of an epidemic event. The findings also offer insights into the development of education and training schemes that allow emergency nurses to acquire and augment their abilities of decision-making and problem-solving in public health responses.

\section{Background}

Over the recent decades, the appearance of emerging infectious diseases (EIDs) with pandemic potential has been accelerating because of the intensifying global commerce activities as well as the widespread disruption of ecological systems [1]. Despite the enormous amount of efforts channelled into advancing mechanisms and technology in infection prevention and control, EIDs continue to represent a substantial health threat to the public health, posing an explicit challenge to both developed and developing countries [2]. To tackle the long-lasting pandemic threats towards people worldwide and ensure global health security, it is of paramount importance for international communities and organizations to collaborate and coordinate the global endeavours in enhancing public health surveillance and response capacities [3].

Allied with the global endeavours to combating the spread of EIDs and the occurrence of epidemics is the local efforts of healthcare systems and healthcare institutions. To improve healthcare facilities' capacity 
to meet possible challenges during an EID outbreak, the World Health Organization (WHO) has formulated a preparedness checklist for potential outbreaks [4]. The checklist portrays the structure of an incident command system, assisting healthcare administrators to gain ground on efficient functioning in a hospital-based response to an outbreak. To strengthen the preparedness and response planning of local healthcare systems, the checklist offers advice for the establishment of a network of healthcare facilities, which consists of public hospitals, private hospitals, professional medical organizations, and other non-governmental organizations [5]. This network would coordinate the multi-sectoral collaboration of healthcare system units and departments in preparing for and responding to an EID event, in the aspects of disease surveillance, infection control, quarantine, treatment, and prophylaxis [6].

The capacity to potential outbreak respond involves healthcare institutions and facilities not only drawing up contingency plans to meet the precautionary needs required, but also preparing and equipping frontline healthcare providers for impending disease situations [7]. Within the healthcare system, accident and emergency departments (AEDs) are directly available to the public and serve as the main gateway to the delivery of healthcare services, and emergency nurses therefore have often been at the forefront of outbreak response [8]. Indeed, they are involved in various duties during the management of an epidemic event, such as early recognition of suspected or infected patients, implementation of proper infection control measures, and coordination of patient logistics [9].

However, it is reported that emergency nurses could be confronted with various difficulties in the process of EID management. For instance, one of the major challenges emergency nurses faced amid an EID outbreak is the elevated but unpredictable risk for infection. The risk for exposure to infected patients in the emergency departments was considered to be greater than healthcare professionals from other disciplines during an epidemic event, as the infectious status of the attendance of emergency departments are mostly undiagnosed and unconfirmed [10]. In addition to the occupational risk for EID infection, another issue encountered by emergency nurses that has been repeatedly reported in the literature was the changes of existing guidelines and recommendations that occur in addressing a particular EID. Studies have highlighted that emergency nurses were required to adjust their practice frequently and rapidly to address the amendments in infection control guidelines, while such amendments could be subtle and implicit $[11,12]$. It is suggested in the literature that the antecedents of these challenges could be consequential to the uncertainty and changes experienced by emergency nurses in the workplace during an outbreak, suggesting that that changes and uncertainty within the work environment is one of the major and primary barriers to emergency nurses practice [13]. While the uncertainty and changes encountered by emergency nurses have been revealed in the literature [14, 15], there has been a paucity of understanding about emergency nurses' actions and strategies to overcome the barriers and address the instabilities and vulnerabilities of the circumstance. Addressing such gaps might offer insight into the development of plans and interventions for promoting the preparedness and response of emergency nurses in prevailing EID events. Thus, the present study aimed to explore emergency nurses' behaviours and strategies in addressing the challenges of uncertainty and change during an EID event. 


\section{Methods}

\section{Design}

The present article serves to depict the data from a larger doctoral project pertaining to the discovery of the process of how emergency nurses engage in the management of EIDs. The present study focuses on illustrating data regarding the behaviours and strategies of emergency nurses in tackling challenges originated from uncertainty and change at the workplace during an EID event. The Consolidated Criteria for Reporting Qualitative Research (COREQ) guidelines were applied in the reporting of the present study [16].

Given the nature of the aim of the research, a qualitative study was set up to explore emergency nurses' perceptions and experiences in the events of epidemics. A grounded theory approach [17] was selected to guide the interactive process of data collection and analysis. This approach places a priority on the interpretation of roles and interactions among individuals within the embedded realities of a phenomenon, which offers insights into the elements that shape their beliefs and behaviour patterns [18]. Although the aim of the present study is not to sophisticate or establish a substantive theory to elucidate emergency nurses' behaviours amid EID events, the grounded theory approach for data collection and analysis is considered to be relevant in the present study. By adopting such an approach to guide data collection and analysis scheme, the interactions between emergency nurses and their situated work environment over the course of EID management were discovered, facilitating an understanding of the actions and strategies they adopted in overcoming the challenges of uncertainty and changes at work.

The Straussian framework of grounded theory data collection and analysis was adopted in the present study. This framework offers explicit and well-defined analytical steps for data analysis with the provision of a coding paradigm, facilitating the discovery of associations among conditions, interactions, and consequences [17]. The detailed framework of data interpretation supports the procedural operations of the data analysis process, which might help to establish the plausibility and completeness of the findings while preserving the intertwined and dynamic nature of the data [19]. Thus, the Straussian framework was employed to help address the inherent aim of data collection and analysis of the present study, which was to gain an in-depth comprehension of emergency nurses' perspectives within the social interaction process of EID management.

\section{Participants}

In line with the grounded theory approach, the recruitment of participants in the present study consisted of a combination of purposive sampling and theoretical sampling strategies. At the beginning of sampling, a purposive sampling scheme was employed to recruit the first 10 participants. This allows for the selection of participants among the relevant population according to an initial set of criteria [20]. Criteria for selecting the participants were as follows: (1) fulltime registered nurse in emergency departments in Hong Kong; (2) Cantonese and English-speaking; and (3) willing to take part in the study to share their experiences. In the later phase, participants were solicited through a theoretical sampling 
scheme. This sampling strategy is an iterative process, in which data seeking and eliciting is driven and performed on the basis of concepts that the previous data alluded [21]. It enables the ongoing development and elaboration of preliminary concepts and categories that emerge in the process of data analysis, and thus grants greater representativeness to the findings [22,23]. The recruitment of participants through theoretical sampling continued until data saturation was achieved, in which a repetition of responses was gathered with no new categories emerged from the data [24]. Eventually, 26 emergency nurses participated in the study. The demographic information of the participants is summarised in Table 1.

\section{Data collection}

Data were collected through conducting semi-structured, face-to-face, individual interviews with the participants by the first author (SKKL). Once eligible individuals agreed to participate, they were provided an information sheet explaining the background and objectives of the present study. The details of their involvement in the study were also indicated and described in the information sheet. After confirming the participants understand the nature of the study and their participation, an interview was scheduled with each participant which the locations and time were arranged according to their preferences. Prior to the interview, demographic information of the participants, such as age range, ranking, and years of work experience, were collected with the use of a demographic data sheet. The interviews were audiotaped under participants' permission. An interview guide, which included board and general open-ended questions, was followed to simulate participants thoughts and opinions regarding their experiences in tackling uncertainty and change during epidemics (see Figure 1). For participants who were recruited by theoretical sampling, areas that were of particular interest to the study were highlighted in the interview guide, while modifications were made to those areas for each interview to address the progress of data analysis and category development. A total of 26 interviews were conducted, and the length of the interviews ranged from 55 minutes to three hours.

\section{Data analysis}

In the present study, the process of theoretical sampling, data collection, and data analysis was characterised as a concurrent procedure that took place simultaneously. This indicates that retrieved insights emerged from the interviews during data analysis helped navigate and inform the directions for subsequent data collection and thus, theoretical sampling [18]. Prior to the analysis of data, each audiotaped interview was transcribed verbatim by the first author (SKKL). The transcripts were then checked against the interview tapes to ensure the accuracy of the transcription. Once the transcription was completed, data analysis commenced with the guidance of the three-phase coding framework suggested by Corbin and Strauss [17], namely, open coding, axial coding, and selective coding.

The first step of the data analysis process was open coding. The primary emphasis in this phase is to discover preliminary codes and categories from the data. In the beginning, the textual content of the interviews was read and reread several times by the first author (SKKL) to capture a general understanding of the participant's points of view. After that, each transcript was scrutinised in detail by 
examining the content line-by-line and paragraph-by-paragraph. Concepts and meaning in the passages that were considered to be of analytical relevance were coded. The codes were further interpreted by the constant comparative method that existing codes were compared with codes emerged from preceding analyses [19]. Those shared similarities in features were collated to form categories.

In the axial coding phase, established categories were refined through examining their connections with each other. In addition to the constant comparative method, the coding paradigm offered by Corbin and Strauss [17] was utilised as an analytic tool to facilitate the exploration of relationships among categories. The coding paradigm highlights four main components for the establishment of connections among categories, which are phenomena, conditions, interactional strategies, and consequences. Related categories were connected and further developed into more sophisticated categories that consisted of clusters of categories and sub-categories.

The aim of the third phase, selective coding, was to identify the core category that was able to underpin the central essence of the phenomenon of inquiry. The core category is characterised by several properties, such as frequent appearance in the data, a considerable degree of abstraction, and extensive connections with all the other categories and codes [17]. In the present study, the determination of the core category was discussed among the authors, and a consensus was made on deciding Rehearsing for improvisation to be the core category that was capable to represent the whole phenomenon under study.

\section{Results}

Rehearsing for improvisation was identified as the core category, delineating how emergency nurses thrived in uncertainty and change in various areas of their practice during epidemics. Four interplaying sub-categories were identified: (1) sensing the unclear situation, (2) equipping for the impending battle, (3) calibrating to the evolving guidelines, and (4) navigating the new role and duties. These categories present the emergency nurses' strategies in addressing uncertainty and change while engaging in EID management.

\section{Rehearsing for Improvisation}

While it may seem oxymoronic to describe a behaviour of rehearsing for improvisation, the findings revealed that emergency nurses were required to prepare and equip themselves with skills and techniques to improvise appropriate decisions and actions in addressing changes and uncertainty during an epidemic. In this circumstance, everyday duties posed challenges to their physical, emotional, and psychological capacity to adapt. The comment below illustrates how an experienced emergency nurse responded to untoward incidents when engaged in EID management:

Various unexpected issues that demand our action come all of a sudden, and we are unable to stop or control them. At this moment, it is time to examine our ability to stand the test of these challenges. It tests our leadership, our problem-solving skills, and our ability to improvise. In addition, it challenges our 
critical thinking skills and decision-making abilities. These are all crucial as we work in the accident and emergency department, especially in the midst of unpredictable and unforeseen events. (P16)

This view was echoed by another participant, who indicated that technical solutions were inadequate and unavailable for emergency nurses to handle unexpected issues while engaged in EID duties. The participant remarked that it was crucial to identify alternatives and respond to issues on an impromptu, rather than on a prepared basis:

It could be chaotic and problematic in managing EID. We are not able to predict what is ahead waiting for us to handle. Things can happen in a way that is poles apart from what is written in the protocol. (P14)

This participant went on to share his experience in an incident during the period of the Ebola virus disease outbreak, while a patient with suspected infection displayed uncooperative and aggressive behaviour:

There was once a suspected Ebola case transferred to our department via ambulance. The patient had developed signs of infection, therefore we considered him to be high risk and arranged an isolated room for quarantine purposes. But then the patient started to be uncooperative and aggressive, perhaps because of communication problems, as the patient was from an ethnic minority and there was no interpreter available at that moment. Suddenly he turned violent and assaulted our staff and we had to subdue and restrain him, while we had no time to gown up in PPE. At that time, we were so helpless and we were afraid of being infected. The guidelines and protocols did not mention what we could do in this situation, and we had to count on ourselves. (P14)

This incident showcased a situation emergency nurses encountered while engaging in EID management of an unpredictable and unexpected event, as described by some participants, that "stirred up troubles". Instead of following established protocol, emergency nurses were required to prepare and equip themselves with skills and strategies to improvise and adapt in such a situation.

\section{(1) Sensing the unclear situation}

\section{Some participants expressed the belief that the most pertinent way to resolve uncertainty was to obtain relevant information to address any erratic situations. Indeed, gathering up-to-date information was considered by participants to be a crucial component for emergency nurses to acquire a}




\section{general picture of the nature and progress of an EID scenario, enabling them to orientate themselves to the circumstances. A participant highlighted the importance of obtaining relevant information when gaining familiarity with an EID scenario as follows:}

It is of the utmost importance that you know what is happening. As long as you understand the situation, you realize the problem. You have to acquire the latest information and maintain an up-to-date understanding of the situation. (P16)

One of the major concerns raised by the participants was about the quality of the information, as some of them pointed out that the information they received was not standardized. Participants stated that the information provided by their colleagues, which included disease information, infection control guidelines, and patient logistics protocols, was sometimes inconsistent, leading to confusion. Although they worked in two different hospitals, these two participants held similar opinions about the inconsistency of the information they received. One of the participants described the problem as follows:

The information could sometimes be regarded as 'hearsay'. Perhaps one staff member had said something about the disease, then others started to discuss and circulate the information. However, no one had confirmed the creditability or sources of that piece of information. The information might be distorted, exaggerated, or even misleading. However, we do not have an official and standardized source for obtaining information, and therefore hearsay persists among staff. (P20)

While facing issues such as information overload and inconsistent information, participants highlighted that personal alertness and vigilance were also required in addressing the unclear situations they were facing, instead of depending entirely on provided information. In their everyday work, emergency nurses served as gatekeepers who were closely connected to the community, which helped provide clues as to the disease trend and progress and allowed them to reveal the development of the general disease situation. The comment below illustrates how a participant recognized the outbreak of H1N1 influenza through engaging in routine practice:

You know about the disease situation and progress at work, especially if you are the triage nurse. There was a large number of patients attending AED and eight out of 10 had similar flu-like symptoms, you would then realize and be able to tell, there was something wrong, it was the influenza that was causing this. Then you read the news, and took note of the announcement from the Department of Health about recent outbreaks, and you would be alert as to what you had just come across at work. So, you started to pay attention to the outbreak-affected regions, the mortality rate and the prognosis. You experienced it and sensed it. This sense did not merely improve your alertness, but also provided you with the whole picture of the outbreak, including the severity, the magnitude and the extent. (P17) 


\section{(2) Equipping for the impending battle}

\section{Participants referred to EID management amidst}

uncertainty as analogous to a "battle", implying that it was an immense hardship for them and they were required to prepare beforehand and be wellequipped to conquer the battle against EIDs. It was suggested by the participants that they had to equip themselves with relevant knowledge and skills, in order to bolster their readiness both theoretically and practically, to respond to an epidemic event. For instance, one participant, who had extensive experience in emergency care, suggested that holding the relevant knowledge of an EID was a basic necessity for their participation in an outbreak response:

Sometimes there are junior colleagues making mistakes in simple tasks while handling EID cases. The main reason is that they are not familiar with this type of knowledge. I often ask them to do some infectious diseases revision. This is basic for emergency nurses. For example, being able to identify the signs and symptoms of an EID is the most important task in EID management, but if the nurse did not have the related knowledge, how can one differentiate infected patients from the others? (P17)

Indeed, engaging in EID event management required emergency nurses to demonstrate proficiency in a variety of skills and techniques. The participants reported that this specific skill set, which included but was not limited to clinical assessment skills and precautionary measures, enabled them to accomplish various unforeseeable tasks in an effective and proper manner while engaged in EID management. An advanced practice nurse highlighted the necessity of emergency nurses to develop the skill of rapid and accurate clinical assessment for patient surveillance as follows:

An accurate primary assessment is the basic skill an emergency nurse should have, especially at the initial triage. It is very important for us to really learn and be proficient in this skill, because on some occasions, we only have a few seconds to make a triage decision. For example, imagine I am the triage 
nurse, and you are a patient walking towards me. While you are walking, I am already assessing your condition, from your gait, your power, your posture, and your breath. Combined with a patient's past health history, vital signs, and chief complaints, I will have a preliminary picture of their condition. Treatment could then be initiated if needed. (P21)

Considering the importance of obtaining pertinent knowledge and skills, there was a variety of sources for emergency nurses to receive relevant education and learn the required techniques for engaging in EID management, such as workshops and drills. These training, as described by the participants, offered opportunities to AED staff to familiarize themselves with the process and procedure of managing epidemic events, which were not certain but likely to occur. One participant shared her experience of an Ebola drill, commenting as follows:

There was an Ebola drill in our department not long ago. We participated in a simulation for the admission of a patient with confirmed Ebola infection into the AED. It started from the very beginning, from receiving a phone call from the ambulance for the admission of the Ebola case, to triage, to the treatment and arrangement. Staff were assigned to different roles in the drill, such as triage nurse and nursing officer in-charge. We can learn and reinforce the importance of infection control through the drill. We will know how to manage this kind of contingency if they really encounter such an issue in the future. (P16)

Indeed, participants agreed that conducting training in a simulated environment offered a platform to assist emergency nurses in preparing for an epidemic event, and in enhancing their performance to respond to an EID scenario. Yet, the participants highlighted that it was also necessary for emergency nurses to accumulate clinical experience to establish their readiness and proficiency in EID management, which was irreplaceable, and would not be possible by only doing drills and receiving instruction. As asserted by the participants, prolonged engagement in clinical settings offered a benefit to emergency nurses, because clinical wisdom could only be developed by immersing themselves in the substantial aspects of everyday practice. The comment below illustrates a participant's opinion on the significance of accumulated clinical experience on establishing readiness in managing an unanticipated situation:

In the management of EIDs, there are enormous unexpected issues that one might have no idea about how to handle, unless one had accumulated clinical experience or there were others who could share their own experience. It would be difficult for a junior emergency nurse, who had never come across similar issues in reality, to figure out a solution, even if he had attended workshops or drills before. Only if he had actually experienced this type of issue before, could he identify the possible difficulties that might emerge. Then he would have learned from the experience and be ready to handle similar situations in the future. (P22)

\section{(3) Calibrating to evolving guidelines}


The development of an EID situation could wield enormous influences on the implementation of precautionary measures. Among the various indicators depicting the progress of an EID situation, the enacting of the three-tier response level (Alert, Serious, and Emergency) was regarded by participants as the most decisive consideration for their hospitals and departments in stepping up the requirements for routine infection control practice. Stringent infection control recommendations were established to tie in with the exacerbation of the disease situation. Most participants valued the relevance of these accelerated infection control measures in protecting both healthcare personnel and the public from EIDs. However, the changes also impacted emergency service delivery. Some participants did not feel confident about their readiness to adhere to the guideline changes due to lack of practice, although instructions were provided. They commented that there were distinct differences between the new recommendations and the practices they had been accustomed to following, which caused additional complications in successfully implementing the new recommendations. One participant expressed 


\section{concerns about adhering to the updated PPE guidance in an Ebola response, depicting an example of the challenges emergency nurses might encounter in terms of guideline changes:}

There are new recommendations on the standard of the PPE kits for use in handling patients with suspected or confirmed Ebola infection, including a new gown, thicker gloves, additional rain boots, and an extra hood. It is different from the one we are used to using when dealing with other infectious diseases. Not only the equipment, but also the methods and sequences in equipping and removing the gear are totally new to us. Although we have been told and taught in workshops how to utilize the new $P P E$, it is still difficult for us to be readily familiar with the new recommendations without an opportunity to practice. $(\mathrm{P} 17)$

With regard to the implementation of new measurements and protocols, participants had underlined the significance of the idea of calibration, in which new guidelines and recommendations were scrutinized, selectively extracted and adjusted to fit the particular situation in practice. The comment below illustrates how a participant described the process of calibration:

Learning from new guidelines and recommendations is like peeling an onion - you peel off something layer by layer. What is left behind is something that could be incorporated into clinical practice. We should understand that what we have learned or adopted formerly is valuable, and what we are doing now is renewing it to fit the situation. We do not have to do everything by the book. Instead, we should adapt to the process, and adapt to the situation. (P26)

Indeed, many participants suggested that it was of paramount importance to value both their experience and the new recommendations through calibration. They might develop a tailored set of practices through integrating the new recommendations into their experience, enabling them to adapt to the novel needs of EID management. One participant illustrated his experience in adapting to the changes in routine as follows:

In handling a new situation, coping with new challenges, your experience is always invaluable. However, it is important to understand the core intentions embedded in the new guidelines and recommendations, and combine what I have learned with the new knowledge. I would filter the new guidelines and apply them alongside my original set of practices, as long as those guidelines do not violate the basic principles of what I have established from my clinical experience. It is the essence of the new guidelines that I should take into account, but not rigidly adhere to any recommendations. (P14)

\section{(4) Navigating the new role and duties}


In facing an EID event, the scope of emergency healthcare services would be broadened with more emphasis on infection prevention and control, on top of the usual life-saving practice of emergency care provision. Although all participants acknowledged emergency nurses' responsibilities in an epidemic event response, some encountered difficulties embodying the extended role in practice. Some participants commented that the responsibilities and duties of their role as an emergency nurse had changed when they were engaged in EID management. They suggested that adjusting to the altered role was challenging, because there was a lack of clarity surrounding their role and orientation in the midst of an epidemic event. For instance, one participant, who was relatively new to the emergency care setting, expressed concerns about articulating the role expectations that accompanied the responsibilities of an emergency nurse in the H7N9 avian influenza scenario, as follows:

In the course of EID management, I have a feeling that I am not working in an AED. I formerly expected that the role of an emergency nurse was to triage patients according to their conditions and offer care to those who were in critical and urgent need. But now my role has changed all of a sudden and I am mainly assigned to duties on infection prevention. It is true that the guidelines and measures for disease prevention and infection control have been put in place, but the problem is, I personally am not yet in place. (P12) 
On this topic, a participant who served as a department operations manager and was regarded as being in a higher management position, offered an explanation for the instruction of minimal intervention in suspected Ebola cases. She highlighted the importance of maintaining an open mind while facing a significant change in work practices, in the following way:

Ideally, we should do everything we can for patients, and this is what we always do in AEDs. However, we should also consider the outcomes and consequences of our actions and interventions. For patients with Ebola, resuscitation might not do much good for them while at the same time, it might subject our staff to a risk of infection. There are various issues we have to consider when making a decision, and therefore we have to be flexible and remain open on these grounds, rather than sticking to the same old rut all the time. (P26)

A few participants agreed that working adaptively and flexibly was the essential attribute of emergency nurses in navigating their altered role in accommodating evolving needs during EID management. They generally acknowledged and displayed an acceptance of the extended role, and endeavoured to cultivate adaptiveness and assimilate the duty and responsibility of infection prevention into their regular work practices. As one participant put it:

Sometimes one should allow changes to take place and show openness towards the changes. Now, I can say the practice of infection control has seemingly integrated as a usual component into my emergency care practice, regardless of the alert level and disease situation. It is a process that takes time. (P13)

\section{Discussion}

The emergency nurses in the present study demonstrated their prudence to orientate themselves to an ambiguous work situation and displayed the flexibility to embrace changes in their practice, roles, and duties. To address uncertainty, emergency nurses attempt to obtain information regarding an encountered situation, which involves information about the disease and specific guidelines in response. Acquiring precise information during an EID event is essential for nurses, enabling them to obtain relevant facts, such as disease identification, management, and prevention. Also, effective information provision could strengthen nurses' capacity in offering health promotion and health education in the community, which would help quell public fears [25]. Despite the importance of the expeditious provision of epidemic information, the emergency nurses in the present study pointed out that the disease information they received could be excessive and non-standardized, creating confusion and conflicts for them in practice. The problem of information overload during epidemics is in fact reported in previous studies [26, 27], indicating the need for healthcare facility administration and management to review and revise the effectiveness of current information dissemination strategies and systems. Also, healthcare administrators should not merely disseminate information among nurses across services, but also streamline information appropriately in order to facilitate its integration into routine practice.

In addition to obtaining information from official sources, nurses in this study reported that they would gain an overall impression of a disease situation through observation and clinical encounters, as they 
would evaluate a disease situation according to the number of patients who presented with symptoms and the severity of the disease. While such a strategy is seemingly useful for nurses in obtaining firsthand information on an EID event, this might result in inaccurate estimations of the disease situation and adversely influence their awareness of the situation [28]. For instance, in their study, Sridhar et al. [29] reported that healthcare workers might underestimate their likelihood of Ebola infection, because the incidence and seriousness of infection is comparatively lower from their practice experience than infections quoted in the existing data. Consequently, this might undermine their awareness of the disease. Therefore, healthcare facilities should maintain communication with nurses and other frontline healthcare workers to facilitate a more precise estimation of the magnitude of an EID situation. Due to the uncertainties surrounding the workplace environment and the rather provisional and unconfirmed information regarding a given disease situation, emergency nurses are unable to articulate the skills and tactics required to handle relevant EID management tasks in a fully rehearsed manner. The findings in the present study reveal that emergency nurses would uphold improvisational responses in part with limited available information to support their decision-making and practices. These improvisational responses are essential for nurses to expediently address unpredictable or unexpected events in the course of EID management.

Following along with uncertain situations are the changes required to respond to such situations. In the findings, these changes involve the changes in the situation, the changes in the information provided, and the changes that nurses should accordingly be making. Indeed, it is repeatedly cited in the literature that changes in the workplace always create tension for an organization's stakeholders, including those who decided to initiate the change, and those who were required to implement the change [30]. Changes in the general clinical context of EID management would have considerable impact on nurses' usual practice, expectations, and work practices. A change of environment might induce structural changes in the workplace, which might require nurses to change from a well-adapted and accepted behavior or working style to a new and unfamiliar practice. These changes might induce insecurity and create further uncertainty among nurses [31]. The changes might also pose challenges to nurses' practice, as workplace changes often involve possible increases in workload and the likelihood of mistakes being made during practice [32]. This might possibly explain the reluctance of some nurses in the present study to accept the changes in different aspects of emergency care provision during EID management.

As indicated in the findings, some nurses showed their willingness to acclimate to change despite the possible difficulties, as they realized the importance of change in meeting new EID management challenges. However, it was reported that inadequate time and opportunity was allocated to allow them to adjust their practice and incorporate the changes into their practice. This finding is in keeping with previous studies, showing that changes made within healthcare facilities might not always be in line with the ability of healthcare workers to adjust [33,34]. Discrepancies may exist between the expectations of hospital administration on the renewal of existing practices, and the actual preparedness and capacity of workers to adopt the new practices [11]. It is suggested that a prudent approach, sensitive to the overall readiness of nurses in learning new practices or standards, should be employed by healthcare facility administrators to enable adequate training for nurses. 
Although key knowledge and skills in public health and infection control are integrated as a compulsory part of most current nursing curricula [35], the present study's findings point out that emergency nurses are frequently assigned to new roles and are required to perform unfamiliar tasks during an epidemic event, which might well be beyond the scope of their previous practice. For instance, they are required to shoulder the responsibility of public health surveillance in the course of an EID event, including case ascertainment and contact tracing, which could be perceived by emergency nurses as an extra duty outside of their usual domain of practice. In order to strengthen emergency nurses' capacity in subsequent epidemics, education and training should be provided to equip them with the relevant skills, knowledge, and attitudes required for effectively serving in their role and acting accordingly in the unprecedented circumstances of an EID outbreak. Training and education in hospital preparing nurses for EID management often emphasizes updating the technical skills required to implement infection control measures, such as hand hygiene practices and PPE use [36]. What might have been overlooked is training and practice in the acquisition and augmentation of emergency nurses' abilities in terms of decisionmaking and problem-solving. The importance of such abilities is highlighted in the findings. Thus, apart from technical skills, educational and training outcomes should place equal emphasis on developing nurses' cognitive skills, such as critical thinking, calibration, and prioritizing, and on preparing them with the capacity to process and apply knowledge in chaotic and complicated conditions. In addition, emergency nurses' interpersonal skills should be strengthened, to facilitate the establishment of a cohesive work environment that supports healthcare workers in persevering in the face of the intense stress of managing an EID.

\section{Limitation}

The major limitation of the present study is that a substantive theory is not developed in the findings regardless of the use of a grounded theory approach in data collection and analysis. Despite the fact that the findings have established a "conceptual ordering" of well-developed and plausible categories that delineates the properties of emergency nurses' experiences, perceptions, and actions [17], such descriptions are incapable to establish an integrated theoretical scheme. In other words, the core category Rehearsing for improvisation will not be able to offer in-depth explanations or predictions about the phenomenon of how emergency nurses address the challenges of uncertainty and change during epidemics. Notwithstanding this limitation, the present study aptly illustrates the descriptive details, which are empirically grounded in research, pertaining to emergency nurses' behaviours and strategies in combating uncertainty and change in their workplace. These findings could provide useful insights for future recommendations or research on emergency nurses' preparedness and competence in public health responses.

\section{Conclusion}

The findings delineate that emergency nurses were required to prepare and equip themselves with skills and strategies to improvise and adapt to the management of an epidemic event. In addition to factual 
information, emergency nurses would gather their own first-hand information through everyday practice, which assisted them in addressing the situations they encountered. In addressing the duties and responsibilities embedded in their role, it was important for emergency nurses to demonstrate critical thinking and the ability to be adaptive. To reinforce the preparedness of emergency nurses, learning by practical experience might be taken into account as it is considered a proper scope of training for emergency nurses involving in the management of epidemics, while the essence of clinical wisdom could be nurtured throughout the process.

\section{Abbreviations}

EID: Emerging infectious disease; AED: Accident and emergency department; WHO: World Health Organization; PPE: Personal protective equipment.

\section{Declarations}

\section{Ethics approval and consent to participate}

Prior to commencing the present study, ethical clearance of the larger study was sought from and approved by the Human Ethics Committee of the Hong Kong Polytechnic University (no reference number, approved November 2013). Thorough information about the nature of the research and the participation was provided to participants. All the emergency nurses who participated in the study gave their informed written consent regarding their involvement in the study and their permission for audiotaping the interviews. Throughout the research process, the participants' anonymity and confidentiality were guaranteed by various strategies, such as removing any personal information and identifiers in the transcripts, masking their identities by replacing their name with unique codes, and protecting the digital recordings and documents in encrypted files to prevent unauthorised access.

\section{Consent for publication}

Not Applicable.

\section{Availability of data and material}

The datasets used and/or analysed during the current study are available from the corresponding author on reasonable request.

\section{Competing interests}

The authors declare that they have no competing interests.

\section{Funding}

The authors received no financial support for the research, authorship and/or publication of this article. 
SKKL contributed the study design, data collection, data analysis, and led the drafting of the manuscript. EWYK, MSYH, SMCP and WTC contributed to study design, data analysis and the drafting of the manuscript. All authors read and approved the final manuscript.

\section{Acknowledgments}

The authors would like to thank the participants of the present study for sharing their valuable insights.

\section{References}

1. Hoberg EP, Brooks DR. Evolution in action: climate change, biodiversity dynamics and emerging infectious disease. Philos T R Soc B. 2015;370(1665):1-7. https://doi.org/10.1098/rstb.2013.0553

2. Rappuoli R, Black S, Bloom DE. Vaccines and global health: In search of a sustainable model for vaccine development and delivery. Sci Transl Med. 2019;11(497):eaaw2888. https://doi.org/10.1126/scitranslmed.aaw2888

3. Lam SKK, Kwong EWY, Hung MSY, Pang SMC, Chiang VCL. Nurses' preparedness for infectious diseases outbreaks: a literature review and narrative synthesis of qualitative evidence. J Clin Nurs. 2018;27(7-8):e1244-e1255. https://doi.org/10.1111/jocn.14210

4. World Health Organization. Hospital preparedness for epidemics. https://apps.who.int/iris/bitstream/handle/10665/151281/9789241548939_eng.pdf? sequence=1\&isAllowed=y (2014). Accessed 26 May 2019.

5. Rezaei F, Maracy MR, Yarmohammadian MH, Sheikhbardsiri H. Hospitals preparedness using WHO guideline: a systematic review and meta-analysis. Hong Kong J Emerg Me. 2018;25(4):211-222. https://doi.org/10.1177/1024907918760123

6. Singh SR, Coker R, Vrijhoef HJ, Leo YS, Chow A, Lim PL, Hildon, ZJL. Mapping infectious disease hospital surge threats to lessons learnt in Singapore: a systems analysis and development of a framework to inform how to DECIDE on planning and response strategies. BMC Health Serv Res. 2017;17(622):1-14. https://doi.org/10.1186/s12913-017-2552-1

7. Labrague LJ, Hammad K, Gloe DS, McEnroe-Petitte DM, Fronda DC, Obeidat AA, Mirafuentes, EC. Disaster preparedness among nurses: a systematic review of literature. Int Nurs Rev. 2018;65(1):4153.

8. Baduge MSP, Morphet J, Moss C. Emergency nurses' and department preparedness for an Ebola outbreak: a (narrative) literature review. Int Emerg Nurs. 2018;38:41-49.

https://doi.org/10.1016/j.ienj.2017.12.002

9. Lam SKK, Kwong EWY, Hung MSY, Pang SMC, Chien WT. A qualitative descriptive study of the contextual factors influencing the practice of emergency nurses in managing emerging infectious diseases. Int. J. Qual. Stud. Health Well-being. 2019;14(1):1626179.

https://doi.org/10.1080/17482631.2019.1626179 
10. Choi JS, Kim JS. Factors influencing emergency nurses' ethical problems during the outbreak of MERS-CoV. Nurs Ethics. 2018;25(3):335-345. https://doi.org/10.1177/0969733016648205

11. Lam SKK, Kwong EWY, Hung MSY, Pang SMC. Bridging the gap between guidelines and practice in the management of emerging infectious diseases: a qualitative study of emergency nurses. J Clin Nurs. 2016;25(19-20):2895-2905. https://doi.org/10.1111/jocn.13343

12. Zimmerman PA, Mason M, Elder E. A healthy degree of suspicion: a discussion of the implementation of transmission based precautions in the emergency department. Australas Emerg Nurs J. 2016;19(3):149-152. https://doi.org/10.1016/j.aenj.2016.03.001

13. Lam SKK, Kwong EWY, Hung MSY, Pang SMC, Chien WT. Emergency nurses' perceptions of their roles and practices during epidemics: A qualitative study. Br J Nurs. 2019;28(8):523-527. https://doi.org/10.12968/bjon.2019.28.8.523

14. Delgado C, Upton D, Ranse K, Furness T, Foster K. Nurses' resilience and the emotional labour of nursing work: an integrative review of empirical literature. Int J Nurs Stud. 2017;70:71-88. https://doi.org/10.1016/j.ijnurstu.2017.02.008

15. Wolf LA, Perhats C, Delao AM, Clark PR. Workplace aggression as cause and effect: emergency nurses' experiences of working fatigued. Int Emerg Nurs. 2017;33:48-52. https://doi.org/10.1016/j.ienj.2016.10.006

16. Tong A, Sainsbury P, Craig J. Consolidated criteria for reporting qualitative research (COREQ): a 32item checklist for interviews and focus groups. Int J Qual Health Care. 2007;19(6):349-357. https://doi.org/10.1093/intqhc/mzm042

17. Corbin JM, Strauss AL. Basics of qualitative research: Techniques and procedures for developing grounded theory. Thousand Oaks, California : SAGE Publications Inc; 2015.

18. Bryant A, Charmaz K. The SAGE handbook of current developments in grounded theory. London: SAGE Publications Ltd; 2019.

19. Handberg C, Thorne S, Midtgaard J, Nielsen CV, Lomborg K. Revisiting symbolic interactionism as a theoretical framework beyond the grounded theory tradition. Qual Health Res. 2015;25(8):1023-1032. https://doi.org/10.1177/1049732314554231

20. Etikan I, Musa SA, Alkassim RS. Comparison of convenience sampling and purposive sampling. AJTAS. 2016;5(1):1-4. https://doi.org/10.11648/j.ajtas.20160501.11

21. Giles TM, de Lacey S, Muir-Cochrane E. Coding, constant comparisons, and core categories. Adv Nurs Sci. 2016;39(1):E29-E44. https://doi.org/10.1097/ANS.0000000000000109

22. Holloway I, Wheeler S. Qualitative research in nursing and healthcare. Chichester: John Wiley \& Sons Ltd; 2017.

23. Gentles SJ, Charles C, Ploeg J, McKibbon K. Sampling in qualitative research: Insights from an overview of the methods literature. Qual Rep. 2015;20(11):1772-1789. http://nsuworks.nova.edu/tqr/vol20/iss $11 / 5$

24. Aldiabat KM, Navenec L. Data saturation: the mysterious step in grounded theory method. Qual Rep. 2018;23(1):245-261. https://nsuworks.nova.edu/tqr/vol23/iss1/18 
25. Stirling BV, Harmston J, Alsobayel H. An educational programme for nursing college staff and students during a MERS-coronavirus outbreak in Saudi Arabia. BMC Nurs. 2015;14:20. https://doi.org/10.1186/s12912-015-0065-y

26. Belfroid E, Timen A, van Steenbergen JE, Huis A, Hulscher, ME. Which recommendations are considered essential for outbreak preparedness by first responders? BMC Infect Dis;2017:17(195):111. https://doi.org/10.1186/s12879-017-2293-0

27. Lam KK, Hung SY. Perceptions of emergency nurses during the human swine influenza outbreak: a qualitative study. Int Emerg Nurs. 2013;21(4):240-246. https://doi.org/1016/j.ienj.2012.08.008

28. Goodband A, Oakley S, Rayner J, Toms J, Brostoff J. Influenza: disease, epidemiology and the importance of vaccination uptake by healthcare workers. Prim Health Care. 2014;24(7):33-38.

29. Sridhar S, Brouqui P, Fontaine J, Perivier I, Ruscassier P, Gautret P, Regner, I. Risk perceptions of MSF healthcare workers on the recent Ebola epidemic in West Africa. New Microbes New Infect. 2016;12:61-68. https://doi.org/10.1016/j.nmni.2016.04.010

30. Daiker BL. Adaptive challenges in medical practices. J Med Pract Manage. 2013;28(6):332-340.

31. Corazzini K, Twersky J, White HK, Buhr GT, McConnell ES, Weiner M, Colón-Emeric, CS. Implementing culture change in nursing homes: an adaptive leadership framework. Gerontologist. 2014;55(4):616627. https://doi.org/10.1093/geront/gnt170

32. Maclntyre CR, Chughtai AA, Seale H, Richards GA, Davidson PM. Uncertainty, risk analysis and change for Ebola personal protective equipment guidelines. Int J Nurs Stud. 2015;52(5):899-903. https://doi.org/10.1016/j.ijnurstu.2014.12.001

33. Maddigan J, Butler M, Davidson J. Changing nursing practice: Implementation challenges of intentional rounding on three rehabilitation units. Healthc Manage Forum. 2019;1-5. https://doi.org/10.1177/0840470419849612

34. Sharma N, Herrnschmidt J, Claes V, Bachnick S, De Geest S, Simon M. Organizational readiness for implementing change in acute care hospitals: an analysis of a cross-sectional, multicentre study. $\mathrm{J}$ Adv Nurs. 2018;74(12):2798-2808. https://doi.org/10.1111/jan.13801

35. Clark M, Raffray M, Hendricks K, Gagnon AJ. Global and public health core competencies for nursing education: a systematic review of essential competencies. Nurse Educ Today. 2016;40:173-180. https://doi.org/10.1016/j.nedt.2016.02.026

36. Ellingson K, Haas JP, Aiello AE, Kusek L, Maragakis LL, Olmsted RN, VanAmringe M. Strategies to prevent healthcare-associated infections through hand hygiene. Infect Control Hosp Epidemiol. 2014;35(8):937-960. https://doi.org/10.1086/677145

\section{Tables}

Table 1. Demographic information of the participants 


\begin{tabular}{|c|c|c|c|c|}
\hline $\mathrm{P} 1$ & $\mathrm{M}$ & $25-30$ & Registered Nurse & 7 \\
\hline P2 & M & $25-30$ & Registered Nurse & 7 \\
\hline P3 & M & $25-30$ & Registered Nurse & 2 \\
\hline P4 & $\mathrm{F}$ & $30-35$ & Registered Nurse & 9 \\
\hline P5 & $\mathrm{F}$ & $25-30$ & Registered Nurse & 5 \\
\hline P6 & M & $45-50$ & Nursing Officer & 20 \\
\hline P7 & $\mathrm{F}$ & $20-25$ & Registered Nurse & 1 \\
\hline P8 & $\mathrm{F}$ & $30-35$ & Registered Nurse & 9 \\
\hline P9 & M & $20-25$ & Registered Nurse & 1 \\
\hline P10 & $\mathrm{F}$ & $25-30$ & Registered Nurse & 6 \\
\hline P11 & M & $20-25$ & Registered Nurse & 3 \\
\hline P12 & M & $20-25$ & Registered Nurse & 3 \\
\hline P13 & M & $30-35$ & Registered Nurse & 15 \\
\hline P14 & $\mathrm{M}$ & $30-35$ & Advanced Practice Nurse & 12 \\
\hline P15 & M & $25-30$ & Registered Nurse & 5 \\
\hline P16 & M & $45-50$ & Nursing Officer & 20 \\
\hline P17 & $\mathrm{F}$ & $35-40$ & $\begin{array}{l}\text { Registered Nurse } \\
\text { Page } 21 / 23\end{array}$ & 15 \\
\hline
\end{tabular}




\begin{tabular}{|c|c|c|c|c|}
\hline P18 & $\mathrm{F}$ & $30-35$ & Registered Nurse & 10 \\
\hline P19 & $\mathrm{F}$ & $35-40$ & Registered Nurse & 10 \\
\hline P20 & M & $35-40$ & Registered Nurse & 15 \\
\hline P21 & $\mathrm{F}$ & $35-40$ & Advanced Practice Nurse & 15 \\
\hline P22 & $\mathrm{F}$ & $25-30$ & Registered Nurse & 6 \\
\hline P23 & $\mathrm{F}$ & $45-50$ & Ward Manager & 25 \\
\hline P24 & $\mathrm{F}$ & $35-40$ & Registered Nurse & 15 \\
\hline P25 & $\mathrm{F}$ & $35-40$ & Registered Nurse & 9 \\
\hline P26 & $\mathrm{F}$ & $50-55$ & Department Operations Manager & 30 \\
\hline
\end{tabular}

\section{Figures}

Figure 1. The interview guide

- Please describe your experience as an emergency nurse during epidemics

- Please share with me the possible sources of uncertainty in the workplace.

- Please share with me the possible sources of change in the workplace.

- Please share with me in what way do uncertainty and change in the workplace influence your works and practices.

- Please share with me the methods you use to address uncertainty and change in the workplace. How do you come up with these methods?

\section{Figure 1}

The interview guide 


\section{Supplementary Files}

This is a list of supplementary files associated with this preprint. Click to download.

- supplement1.pdf 